\title{
Characterization of additives in NR formulations by TLC-IR (UATR)
}

\author{
Lidia Mattos Silva Murakami1,2, Joyce Baracho Azevedo1,2, Milton Faria Diniz ${ }^{3}$, Leandro Mattos Silva ${ }^{4}$ and \\ Rita de Cássia Lazzarini Dutra ${ }^{1 *}$
}

\author{
'Instituto Tecnológico de Aeronáutica, São José dos Campos, SP, Brasil \\ ${ }^{2}$ Tenneco Automotive Brasil, Cotia, SP, Brasil \\ ${ }^{3}$ Divisão de Química - AQI, Instituto de Aeronáutica e Espaço - IAE, São José dos Campos, SP, Brasil \\ ${ }^{4}$ Petroquímica Brasken S.A., Capuava, Santo André, SP, Brasil \\ *ritacld@ita.br
}

\begin{abstract}
It is a well-established fact that rubber accelerator is essential to provide solution in different sectors. However, there is a reversal process which can reduce the material performance. Sulfur accelerators donors and organic peroxides have been presented as a solution to the problem. The methodology development that can separate or characterize those components is a challenge and still allows gaps, explained by the application of conventional technique to reach this goal. This study aimed at contributing to the use of off-line coupling of thin layer chromatography (TLC)/infrared spectroscopy (IR) by Universal Attenuated Total Reflection (UATR) for analysis of N-cyclohexyl-2-benzotiazolsulfenamide (CBS), tetraethylthiuram disulfide (TMTD) and dicumyl peroxide (DCP), in natural poly-cis-isoprene (NR) formulations, containing naphthenic oil. The best results were obtained for the plasticizer and DCP, in formulations that had a greater proportion of these compounds. The separation of CBS and TMTD was made with less effectiveness, due to bands overlapping.
\end{abstract}

Keywords: additives, characterization, NR, TLC, UATR.

\section{Introduction}

The competitive industrial environment has demanded of companies more agility in terms of response to the market. Companies have embraced some strategies to accomplish that specific objective and knowing better their own product substantially is one of them. Not only does this route lead to better process variable evaluation, but also it improves the employment of technology.

Products based on polymeric materials play a major role in Aerospace and Automotive Industry considering they can withstand harsh environments, reduces production costs and offers flexibility in processing. As a light solution, the polymers successfully replace metals and traditional compounds due to its mechanical resistance, corrosion resistance and ease in the manufacture of parts with narrower tolerance.

A vulcanized rubber is a widely applied polymer throughout several industrial areas. Its counterpart Natural rubber (NR) for instance, is applied in the automotive industry, like tires and hoses ${ }^{[1]}$, and in aerospace industry, as flexible joints ${ }^{[2]}$. It contains elastomer, vulcanization agent and accelerator, reinforcing filler, stabilizers, among other additives. This mixture is vulcanized after passing through thermal processes or after been exposed to high energy radiation ${ }^{[3]}$. Among these additives, it is possible to highlight the accelerators, which reduce the cure time and consequently the process costs. They are classified according to their chemical composition and / or their speed of action in vulcanization. In Table 1, a list of the most common accelerators $^{[4]}$ is included.

Acknowledge the accelerators and vulcanizing agent of a formulation is paramount for new polymers development. Not only does it aid in reducing process costs, but also it increases the solution spectrum for industry. Taking this into account, the reversion problem is brought to our attention, when the polysulfide bond breaks causing reduction of crosslink density, changing the distribution of the bonds types and modification in main chain structure. These facts lead to reduced article performance.

Reversion resistance of rubber compounds has been obtained by controlling the sulfur content in the crosslink bonds, by applying sulfur donor accelerators and organic peroxides. In terms of thermal stability, their higher bond strength gives more stability than the carbon/sulfur/carbon bond and gives good properties for aging resistance ${ }^{[5]}$.

Study of sulfur vulcanization has been mentioned in the literature $^{[6]}$. TMTD, among others accelerator polysulfides such as N,N-pentamethylenethiuram disulfide (CPTD) were employed and it was concluded that CPTD and its polysulfides are thermally less stable than is TMTD.

As reported by Joseph et al. ${ }^{[7]}$, among the various organic sulphur containing compounds, TMTD has been the most studied. Vulcanizates obtained using this compounds in combination with $\mathrm{ZnO}$ have superior thermal and oxidative 
Table 1. Most usual accelerators and vulcanizing agent for elastomeric compositions.

\begin{tabular}{cc}
\hline Acronym / nomenclature & Acronym / nomenclature \\
\hline MBT (2-mercaptobenzothiazole) & ZDBC (Dibutyldithiocarbamate Zinc) \\
MBTS (benzothiazole disulfide) & ZDEC (zinc diethyldithiocarbamate) \\
CBS (N-cyclohexyl-2-benzothiazolesulfenamide) & ZDMC (zinc dimethyldithiocarbamate) \\
TBBS (N-tert-butyl-di (2-benzothiazolesulfenamide) & DPG (N, N'-diphenyl guanidine) \\
MBS (2- (4-Morpholinothio) benzothiazole) & DOTG (Diortotolylguanidine) \\
TMTD (Tetramethylthiuram Disulfide) & DTDM (4,4'-dithiomorpholine) \\
TMTM (Tetramethylthiuram Monosulfide) & ETU (2-mercaptoimidazoline)/ (ethylene thiourea) \\
TETD (tetraethylthiuram disulphide) & DCP (Dicumyl peroxide) \\
\hline
\end{tabular}

stability and negligible modulus reversion. According to radical mechanism, at vulcanization temperatures, interactive recombination will lead to the formation of accelerator polysulphides (TMTPs).

Influence of sulfenamide accelerators, such as CBS, on cure kinetics and properties of NR has been studied as well ${ }^{[8]}$. CBS accelerator shows the fastest sulfur vulcanization rate and the lowest activation energy (Ea) because CBS accelerator produces higher level of basicity of amine species than other sulfenamide accelerators.

The effect of temperature and peroxide concentration has been studied ${ }^{[9]}$. DCP was evaluated among other peroxides. It was observed that DCP and di-tert-butyl peroxide (DTBP) showed lower amounts of decomposition products compared to other peroxides studied. DCP and DTBP were suggested as better curing agents for NR based rubber compounds at higher peroxide concentrations.

TMTD, CBS and DCP were the additives used in this current paper to be separated and characterized by reason of their chemical characteristics, good properties, including thermal stability and lower content decomposition products.

Fourier Transform Infrared Spectroscopy (FT-IR) is one of the techniques that has been largely applied for materials identification and characterization. However, due to the wide variety of products in the extracts of rubbers and the small concentration of additives contained, it is necessary to perform a separation step by thin layer chromatography (TLC). The association of Infrared Spectroscopy Technique $(\mathrm{TLC} / \mathrm{IR})^{[10,11]}$ is required for chemical structure characterization, although it is hardly used in the literature.

In the TLC/ IR technique, using the $\mathrm{KBr}$ pyramids, the extract is dissolved in the solvent used for the extraction and applied on a suitable silica plate, which is developed in a closed chromatographic vat using a mobile phase (eluent). The plate is dried and the spots revealed, with specific product. The spots are grated and placed in a tube containing the $\mathrm{KBr}$ pyramids. A small amount of solvent used in the extraction is added to conduct the sample to the top of the pyramid. After solvent evaporation, this top is removed, scraped and pressed as a $\mathrm{KBr}$ disc for IR analysis. This technique was started in our laboratories, in the Brazilian Institute, Instituto de Aeronáutica e Espaço (IAE), in $1996^{[10]}$ and it was used for additives analysis, in rubbers ${ }^{[12]}$ as in paints, with positive results ${ }^{[11]}$.

Chauveau et al. ${ }^{[13]}$ separated and identified several vulcanization agents (CBS, MBT, TMTM, DPG, MBT and TMTM) and antioxidants (IPPD and 6PPD) present in hospital rubbers (synthetic polyisoprene-isoprene - IR and copolymer of butadiene and styrene - SBR), by means of the TLC technique. In parallel, the identification of additives by GLC coupled to a mass spectrometer (MS) method was made to confirm the presence or absence of additives.

The samples were extracted in acetone and two types of eluents were used: toluene/ethyl acetate/ammonia $(100 / 5 / 1)$ to extract CBS, MBT, IPPD, 6PPD, TMTM and toluene/acetone/ammonia (45/65/1) to extract DPG, MBT, TMTM. UV fluorescent light was applied to reveal the eluted deposits. It was demonstrated that the TLC method was more efficient in the identification of additives, since it presented greater thermal stability, without degradation of it, during the extraction with acetone at $66^{\circ} \mathrm{C}$. Despite of it, most additives were separated by TLC, even in small amounts of formulations analyzed. and by MS, through fragmented products, there were difficulties in separating some components, such as TMTM and CBS, under the conditions used.

Other recent studies, in IAE laboratories, were carried out to identify additives in polymers using the TLC/IR technique (off-line) and selective extraction. Among them is the Rodrigues et al. ${ }^{[11]}$ publishing, where a painting formulation containing polyurethane (PU) and nitrocellulose (NC) was evaluated. In this study, three eluent systems were used: ethyl acetate/ethyl alcohol (70/35/30), pure toluene and toluene/ethyl acetate (70/30), applying Gibbs developer. Among the four additives of the formulation, it was possible to characterize two of them (ATBC plasticizer and oleamide slider) by UATR.

Damazio et al. ${ }^{[12]}$ applied the same technique, TLC/IR technique (off-line), for the analysis of MBT, TMQ, TMTM and TMTD additives, in ethylene propylene diene monomer terpolymer based rubber (EPDM). Two types of EPDM were evaluated with two different kinds of eluent system: an eluent system with hexane, diethyl ether and acetic acid - 70: 30: 5, and Gibbs developer. The formulation studied contained more than one additive, and thus generated band overlap, but the characteristic absorptions of sulfide additives were revealed by UATR, even though they were in a lower proportion.

Other researches have been done to study rubber additives. For example, the potential of FT-IR analysis of gaseous pyrolyzates (PY-G/FT-IR) for characterization of EPDM additives has also been evaluated ${ }^{[14]}$ TMTM, TMTD, and MBT were employed in this study. Results demonstrated that the PY-G/FT-IR technique can identify additives containing sulfur in concentrations as low as $1.4 \mathrm{phr}(1.26 \%)$ in EPDM. 
However, the method showed some limitation to detect TMTM and TMTD due to overlapping and to similarities of their PY-G/FT-IR spectra, which could not be distinguished from each other.

Although the quoted papers presented favorable results for rubber additives, formulations of NR containing peroxides were not analyzed in comparison with others containing sulfur accelerator or vulcanizing agent systems. There are also limitations for detecting of some sulfur additives. Then, in this paper, the applicability of the TLC/UATR technique was evaluated to characterization of TMTD, CBS and DCP, which are frequently found in NR and EPDM rubbers formulations, used in the industries mentioned earlier. In short, the interest of Automotive and Aerospace Companies in methodology development for problem solving and polymer article improvement is the same.

\section{Materials and Methods}

\subsection{Samples}

The sample of NR was kindly provided by Tenneco Automotive. In Table 2, are presented the formulations developed for NR containing CBS or TMTD or DCP, named, respectively, NR (CBS), NR (TMTD) and NR (DCP).

\subsection{Rubbers preparation}

The raw material was weighed on a precision scale and all components, except for accelerators (TMTD and CBS) and vulcanizing agent (DCP and Sulfur), were mixed in a laboratory banbury 2 liters for $240 \mathrm{sec}$., with a pylon pressure of $4 \mathrm{kgf} / \mathrm{cm}^{2}$, rotation of $70 \mathrm{rpm}$ and initial temperature of $40^{\circ} \mathrm{C}$, to produce the masterbatch. Then it was processed in a roller form six times in an open mill (laboratory cylinder), with rotation of $40 \mathrm{rpm}$. Later, the masterbatch was accelerated in banbury, with the rest of the weighted components (TMTD, CBS or DCP and sulfur) for 120 seconds, with $4 \mathrm{kgf} / \mathrm{cm}^{2}$ pylon pressure, rotation of $70 \mathrm{rpm}$ and initial temperature of $40^{\circ} \mathrm{C}$. Eventually, the rubber was homogenized in roller form six times in an open mill (laboratory cylinder) with a rotation of $40 \mathrm{rpm}$ and removed in blanked.

The rheometer curve interferes in the vulcanization temperature setting of a rubber part. High temperatures can promote a reversal process, where bonds breakage occurs, and consequently the degradation of the polymer ${ }^{[1]}$.

However, the vulcanization times obtained in rheometric tests should be performed at the same temperature as the part will be processed. The value of T 90 should be the time to be used when the material thickness is between 1.5 and $2.5 \mathrm{~mm}^{[1]}$.

Table 2. NR System (phr).

\begin{tabular}{|c|c|c|c|c|}
\hline Components & Functional Group / Structural Formula & $\begin{array}{c}\text { NR } \\
\text { (CBS) }\end{array}$ & $\begin{array}{c}\text { NR } \\
\text { (TMTD) }\end{array}$ & $\begin{array}{c}\text { NR } \\
\text { (PEROXIDE) }\end{array}$ \\
\hline $\begin{array}{l}\text { Natural Rubber } \\
\text { (NR) }\end{array}$ & & 100 & 100 & 100 \\
\hline Naphthenic oil & & 7.7 & 5.4 & 5.4 \\
\hline Zinc oxide & $\mathrm{ZnO}$ & 4.8 & 4.8 & 4.8 \\
\hline Stearic acid & & 1.6 & 1.6 & 1.6 \\
\hline $\begin{array}{c}\text { Carbon Black } \\
\text { N550 }\end{array}$ & $\mathrm{C}$ & 24 & 53 & 53 \\
\hline Sulfur & $\mathrm{S}$ & 2.1 & 3.2 & --- \\
\hline TMTD & & --- & 1.1 & --- \\
\hline CBS & & 1.1 & --- & --- \\
\hline DCP & & --- & --- & 6.4 \\
\hline
\end{tabular}


In this current paper, the compounds vulcanization times were determined from the rheometric curves using a rheometer, from Alpha Technologies, model MDR 2000, at the same vulcanization temperature (T90@160 ${ }^{\circ} \mathrm{C}$ ), and the specimens thickness were $2.5 \mathrm{~mm}^{[1]}$.

For the preparation of vulcanized rubbers slabs, a hydraulic press with vulcanization time of $6 \mathrm{~min}$., temperature in the plateaus of $160^{\circ} \mathrm{C}$ and $150 \mathrm{kgf} / \mathrm{cm}^{2}$ of closing pressure was used.

\subsection{Characterization equipment/conditions}

For the TLC analysis, Merck glass chromatography plates, measuring $20 \times 20 \mathrm{~cm}$ and covered with Silica Gel D60 and glass vat were applied. In the FT-IR analysis, a spectrometer FT-IR Spectrum One PerkinElmer (resolution $4 \mathrm{~cm}^{-1}$, gain 1,4000 to $400 \mathrm{~cm}^{-1}, 20$ scans) was used. FT-IR spectra were obtained, by reflection technique, using the UATR accessory. It was used 20 scans for UATR analysis, based on other papers that they have successfully published for paint ${ }^{[11]}$, rubber ${ }^{[12]}$ and oil analysis ${ }^{[15]}$. This scan number is a suitable to smooth noises in the FT-IR reflection techniques, such as UATR, diffuse reflectance (DRIFT) and attenuated total reflection (ATR).

\subsection{Methodology}

\subsubsection{Extraction and analysis by Infrared spectroscopy (IR) of rubbers and additives}

Vulcanized rubber slabs were cut into small pieces, in sizes of approximately $3.0 \times 3.0 \times 3.0 \mathrm{~mm}$ and placed for extraction in acetone in Soxhlet extractor. The rubber

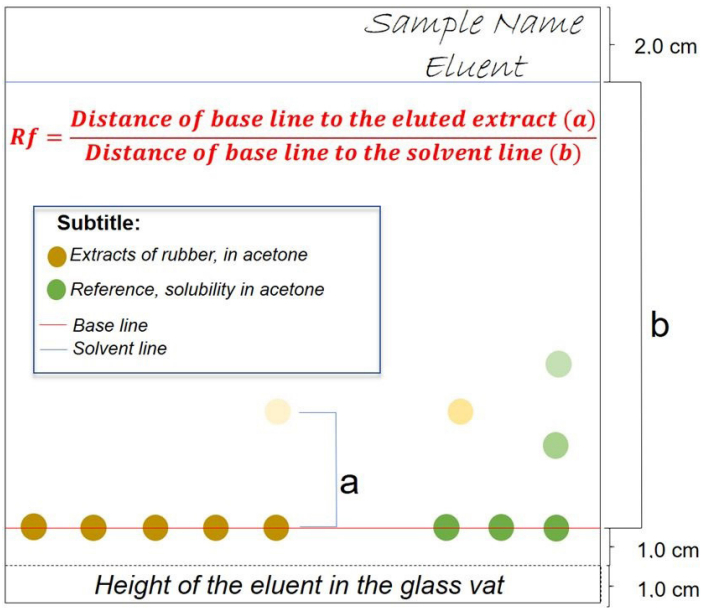

Figure 1. Preparation of the TLC plate for the deposition of the extracts and the RF marking. samples were prepared by pyrolysis (thermal degradation) and analyzed as liquids by transmission, by IR. The extracts containing the additives were analyzed by reflection, UATR.

\subsubsection{TLC analysis}

The TLC plates were labeled and identified with pencils and placed in an oven for 15 minutes at $105^{\circ} \mathrm{C}$ for activation, to remove moisture from the silica. With the aid of a micro syringe, approximately $15 \mu \mathrm{L}$ of extract and pure additives were deposited, side by side, for use as a color reference and retention factor ( $\mathrm{Rf}$ ) on the TLC plate. The additives (TMTD, CBS and DCP), which were powders, were previously solubilized in acetone. The TLC plate was placed in the glass vat and the eluent was added until it reached the $1 \mathrm{~cm}$ mark. The run time of the eluent on the plate was timed until it reached the upper marking of $2 \mathrm{~cm}$. $\mathrm{Rf}$ is the ratio of the distance traveled by the sample to the distance traveled by the eluent in the system (Figure 1).

After running the eluent, excess solvent from the plate was evaporated at room temperature in the exhaust hood, then eliminated in an oven for 15 minutes at $105^{\circ} \mathrm{C}$. Four eluent systems were used, based on literature data: Toluene ${ }^{[16]}$; Toluene/Acetone (45/65) and Toluene/Ethyl Acetate $(100 / 5)^{[13]}$, but in the last two cases ammonia was removed, due it is a product considered to be hazardous to health and the fluorescent developer replaced by the Gibbs reagent. The Toluene/Acetone system (65/45) was also used.

After oven dried and cooled to room temperature, the plates were revealed with a solution of $0.3 \mathrm{~g}$ of the Gibbs reagent (2,6-dichloro-p-benzoquinone-4-chloroimine) in $30 \mathrm{ml}$ of ethyl alcohol. The Rf values and developed colors were noted for each eluted spot which was separated on the TLC plate from extract and pure additives.

\subsubsection{TLC/UATR analysis}

The silica that contained the eluted deposits was scraped washed with $10 \mathrm{ml}$ of acetone and filtered through filter paper on a watch glass. After the eluent evaporation, at room temperature to avoid degrade the material, the samples were analyzed by UATR (Figure 2).

\section{Results and Discussion}

\subsection{UATR analysis of NR extracts, containing different additives}

For the initial evaluation of the additives, extraction was carried out with the appropriate solvent, acetone ${ }^{[10]}$. The extracts were analyzed by UATR as casting films, to avoid the appearance of $\mathrm{KBr}$ moisture bands around 3300 and $1640 \mathrm{~cm}^{-1}$, as a result of solid sample pellets
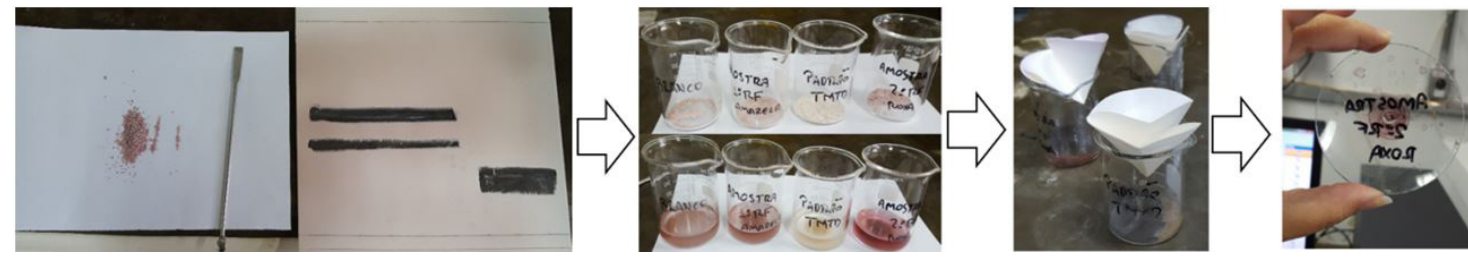

Figure 2. Scraping process of the separated spots by the TLC plate and prepared for UATR analysis. 
preparation, for transmission analysis, that could interfere on the observation of $\mathrm{NH}$ bands of $\mathrm{CBS}$ accelerator.

In Figure 3, it is included the UATR spectra of extracts, in acetone, from the vulcanized NR rubbers samples, compared to additives spectra, taken as references. The objective is verifying if by analyzing of the spectra of NR extracts, it is possible to indicate the different additives presence.

Although the extract spectrum of a rubber shows absorptions of all soluble additives in the used solvent, meaning there are overlapping bands of other formulation additives, it is possible to make some considerations:

- the spectra of Figures 3a, 3c and 3e are different, confirming that the formulations are not the same in terms of organic additives;

- a small number of the major CBS bands, approximately $\left(\mathrm{cm}^{-1}\right)$, assigned to the following functional groups ${ }^{[17,18]}$ : $3200(\mathrm{NH})$ (very low-intensity band, better viewed when the extract spectrum was analyzed separately), 750 (ortho-substituted aromatic ring) and $730\left(\mathrm{CH}_{2}\right)$, is present in the extract spectrum of NR (CBS). However, bands that should appear around 1500 , related to benzothiazole ${ }^{[18]}$, were not visualized. There is also overlapping, in other regions, of naphthenic plasticizer bands, that have aliphatic $\mathrm{CH}$ groups, which absorb at $3000-2900,1460-1400$ and 700-750, and which is in greater proportion, relative to the formulation additives (see Table 2);

- a small number of bands $\left(\mathrm{cm}^{-1}\right)$ around $1240(\mathrm{C}=\mathrm{S}, \mathrm{C}-\mathrm{S})$, $1140(\mathrm{C}-\mathrm{N}), 560(\mathrm{~S}-\mathrm{S})$ characterizes the presence of TMTD ${ }^{[12,17]}$ in the NR (TMTD) extract spectrum. Despite of a greater similarity between NR extract (TMTD) and TMTD spectra that was observed in the fingerprint region, there were overlaps relative to $\mathrm{CH}_{3}$ groups bands between 3000-2900, 1400-1350, present in other additives;

- a small number of bands $\left(\mathrm{cm}^{-1}\right)$ around 1250-1100, 980-870, of variable intensity, weak to strong, assigned to the $\mathrm{C}-\mathrm{O}$ (stronger) and $\mathrm{O}-\mathrm{O}$ (weaker) groups ${ }^{[18]}$, may serve as the basis for characterization of DCP presence. However, this typical intensity variation ${ }^{[12]}$ makes their characterization difficult compared to other additives. Due to other additives band interference in the formulation, it was decided to try to separate the additives, by TLC and TLC/UATR, from the others. It does not mean that the analysis of rubber extracts was already the first step in the methodology to evaluate the indication of different additives presence.

\subsection{TLC analysis of NR extracts and the different additives}

Four eluent systems were used: A) toluene, already used in research on the antioxidant N-phenyl-N'-isopropyl-p-phe nylenediamine (IPPD) in $\left.\mathrm{NR}^{[10]}, \mathrm{B}\right)$ toluene/acetone $(65 / 45)$, C) toluene/acetone $(45 / 65)$ and D) toluene/ethyl acetate (100/5); B, C and D systems being used in our laboratories and in research of different accelerators and antioxidants of NR or poly (cis-isoprene) rubber (IR) ${ }^{[13]}$. Gibbs reagent, already used in other NR research ${ }^{[10]}$, was the developer.

\subsection{NR (CBS)}

In Table 3, TLC data for NR (CBS) rubber are included, using toluene, toluene / acetone (65/45), toluene / acetone $(45 / 65)$, toluene/ethyl acetate (100/5), as eluents, and Gibbs reagent as developer, which will respectively be referred to as: NR(CBS) - Toluene/Gibbs, NR(CBS) - Toluene/acetone (65/45)/Gibbs, NR(CBS) - Toluene/acetone (45/65) / Gibbs and NR(CBS) - Toluene / ethyl acetate (100/5)/Gibbs .

In relation to TLC analysis, only the Toluene/Acetone (45/65) / Gibbs system indicated a possible CBS separation. The other systems did not show efficiency for this procedure, since the colors of deposits and/or RF were different from those observed for respective accelerator. It is apparently caused by the greater plasticizer content and the presence of different products concentrations in the formulation ${ }^{[10]}$ (Table 2, already shown).

\subsection{NR (TMTD)}

In Table 4, is included TLC data for NR(TMTD) rubber, using toluene, toluene/acetone (65/45), toluene/acetone $(45 / 65)$, and toluene / ethyl acetate $(100 / 5)$ as eluents and Gibbs reagent as developer, that will be, respectively, referred to as systems: NR (TMTD) -Toluene / Gibbs, NR (TMTD) -

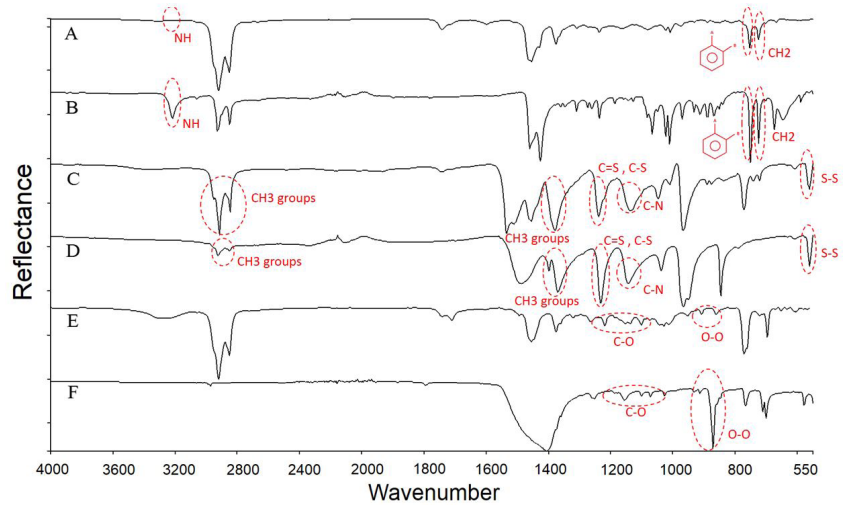

Figure 3. UATR spectra of the acetone extracts of the vulcanized NR, containing additives and references additives: (A) NR (CBS); (B) CBS; (C) NR (TMTD); (D) TMTD; (E) NR (DCP); (F) DCP. 
Table 3. TLC data for NR(CBS) Systems.

\begin{tabular}{|c|c|c|c|}
\hline $\begin{array}{c}\text { Sample } \\
\text { Eluent/Developer } \\
\text { Running time (h: min: sec) }\end{array}$ & Eluted deposit - Color of eluted deposit & $\begin{array}{l}\text { Distance traveled } \\
\text { by the eluent }(\mathrm{cm})\end{array}$ & $\mathbf{R f}$ \\
\hline NR (CBS) & CBS extract - Purple & 8.6 & 0.54 \\
\hline Toluene/Gibbs & NR (CBS) extract $1^{\circ} \mathrm{Rf}-$ Grey & 2.4 & 0.15 \\
\hline 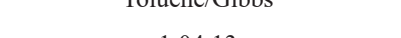 & $\mathrm{NR}$ (CBS) extract $2^{\circ} \mathrm{Rf}$ - Light yellow & 3.0 & 0.19 \\
\hline 1:04:13 & NR (CBS) extract $3^{\circ} \mathrm{Rf}-$ Yellow & 15.0 & 0.94 \\
\hline NR (CBS) & CBS extract - Purple & 14.6 & 0.91 \\
\hline Toluene/Acetone $(65 / 45) /$ Gibbs & NR (CBS) extract $1^{\circ} \mathrm{Rf}-$ Yellow & 14.0 & 0.88 \\
\hline $\begin{array}{c}0: 53: 25 \\
\text { NR (CBS) }\end{array}$ & CBS extract - Yellow & 15.6 & 0.98 \\
\hline Toluene/Acetone $(45 / 65) /$ Gibbs & $\mathrm{NR}$ (CBS) extract $1^{\circ} \mathrm{Rf}$ - Light yellow & 15.5 & 0.97 \\
\hline $\begin{array}{c}\text { 0:48:06 } \\
\text { NR (CBS) }\end{array}$ & CBS extract - Purple & 10.1 & 0.67 \\
\hline Toluene/Ethyl Acetate (100/5)/Gibbs & NR (CBS) extract $1^{\circ} \mathrm{Rf}$ - Light yellow & 2.4 & 0.15 \\
\hline $1: 02: 00$ & $\begin{array}{l}\text { NR (CBS) extract } 2^{\circ} \mathrm{Rf} \text { - Light brown } \\
\text { NR (CBS) extract } 3^{\circ} \mathrm{Rf} \text { - Strong yellow }\end{array}$ & $\begin{array}{l}7.0 \\
7.6\end{array}$ & $\begin{array}{l}0.44 \\
0.48\end{array}$ \\
\hline & NR (CBS) extract $4^{\circ} \mathrm{Rf}$ - Light grey & 14.5 & 0.91 \\
\hline
\end{tabular}

Table 4. TLC data for the NR (TMTD) system.

\begin{tabular}{cccc}
$\begin{array}{c}\text { Sample } \\
\text { Eluent/Developer } \\
\text { Running time (h: min: sec) }\end{array}$ & $\begin{array}{c}\text { Eluted deposit } \\
\text { (Color of eluted deposit) }\end{array}$ & $\begin{array}{c}\text { Distance traveled } \\
\text { by the eluent (cm) }\end{array}$ & Rf \\
\hline NR (TMTD) & TMTD extract - Dark yellow & 3.6 & 0.23 \\
Toluene / Gibbs & NR (TMTD) extract $1^{\circ}$ Rf - Light yellow & 6.6 & 0.41 \\
1:12:44 & NR (TMTD) extract $2^{\circ}$ Rf - Light purple & 8.7 & 0.54 \\
NR (TMTD) & TMTD extract - Dark purple & 13.0 & 0.81 \\
0:46:45 & NR (TMTD) extract $1^{\circ}$ Rf - Light purple & 14.0 & 0.87 \\
NR (TMTD) & & & 0.89 \\
Toluene/Acetone (65/45)/Gibbs & TMTD extract - Yellow & 14.3 & 0.94 \\
Toluene/Acetone (45/65)/Gibbs & NR (TMTD) extract $1^{\circ}$ Rf - Light yellow & 15.0 & 0.99 \\
NR (TMTD) & NR (TMTD) extract $2^{\circ}$ Rf - Light yellow & 15.9 & 0.56 \\
1:00:00 & TMTD extract - Dark purple & 8.9 & 0.56 \\
\hline
\end{tabular}

Toluene/acetone (65/45)/Gibbs, NR (TMTD) - Toluene/acetone (45/65)/Gibbs and NR (TMTD) - Toluene/ethyl acetate $(100 / 5) /$ Gibbs. TLC analysis suggested that is possible to separate this accelerator, especially, by the toluene/ethyl acetate $(100 / 5) / \mathrm{Gibbs}\left(1^{\circ} \mathrm{Rf}\right)$ system.

\subsection{NR (DCP)}

In Table 5, are included TLC data for NR(DCP) rubber, using toluene, toluene/acetone (65/45), toluene/acetone (45/65), and toluene/ethyl acetate $(100 / 5)$, as eluents, and Gibbs reagent as developer, that will be referred to, respectively, as systems: NR (DCP) Toluene/Gibbs, NR (DCP) - Toluene/acetone (65/45)/Gibbs, NR (DCP) - Toluene/acetone (45/65)/Gibbs By the TLC analysis, all the eluent systems showed RF closer to those observed for DCP, although some colors presented differences, possibly because of plasticizer presence, which suggests that the separation is feasible to the accelerator by these eluent systems, with higher accuracy for the Toluene/Acetone (45/65)/Gibbs and NR (DCP) - Toluene/ethyl acetate/Gibbs.

\section{TLC/UATR analysis}

\subsection{NR(CBS)}

By the IR spectra analysis, the separation of CBS presumably did not occur, due to the greater presence of plasticizer (see Table 2). There are only indications of separation beside the evaluation of the spectrum referring to the extract in toluene/acetone (45/65), through bands in $\left(\mathrm{cm}^{-1}\right)$, most likely assigned ${ }^{[17]}$ to the groups: $3329(\mathrm{NH})$, 2922, 2853 and $722\left(\mathrm{CH}_{2}\right)$ and 755 (C-H ortho substitution) (Figure 4).

An interesting fact is under conditions (toluene/Gibbs) for similar formulations of $\mathrm{NR}^{[6]}$, the rubber extract usually produces an eluted deposit, in higher RF and yellow color, which is essentially the plasticizer. Thus, the analysis of the $3^{\circ} \mathrm{RF}$ deposition in toluene was made to characterize the naphthenic plasticizer in the formulation, succeeding in the applied methodology (Figure 5), since that the separated product spectrum $\left(3^{\circ} \mathrm{Rf}\right)$ showed the same absorptions of referred additive. 
Table 5. TLC data for the NR (DCP) system.

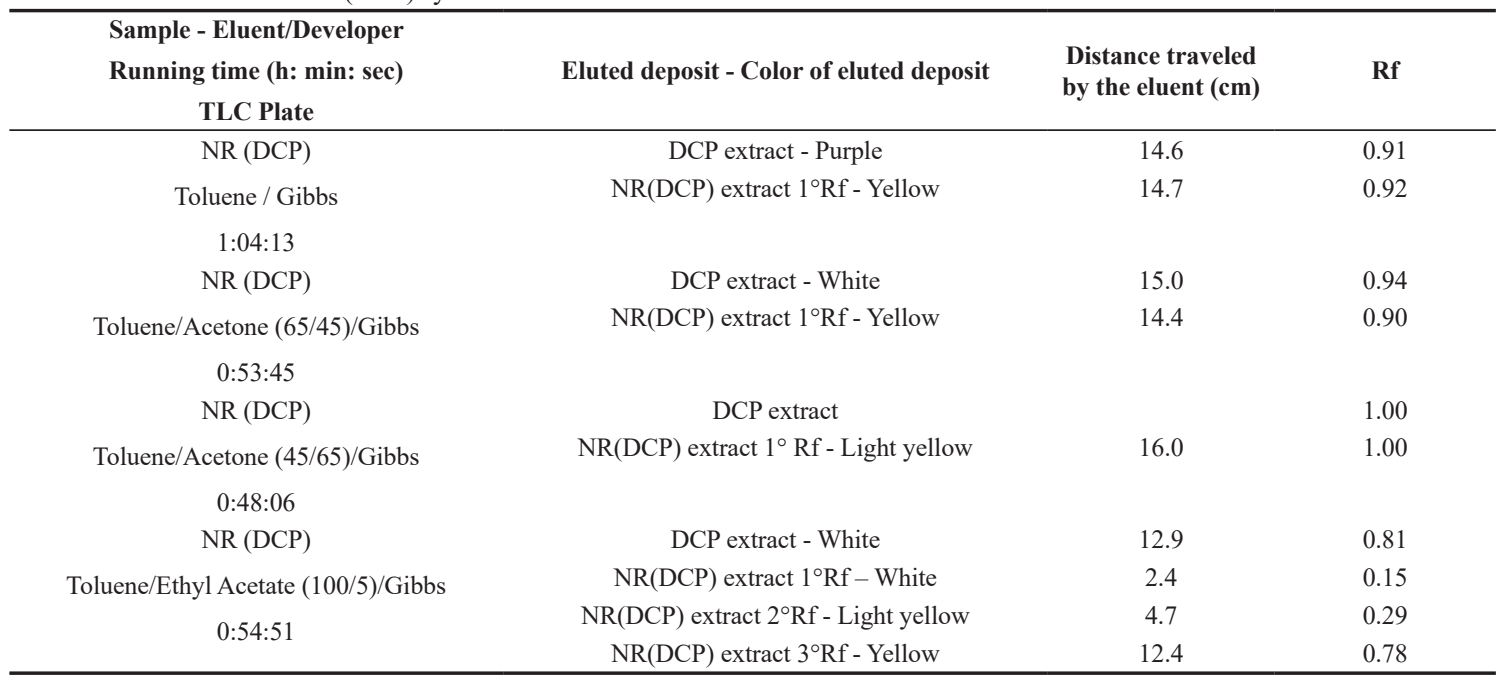

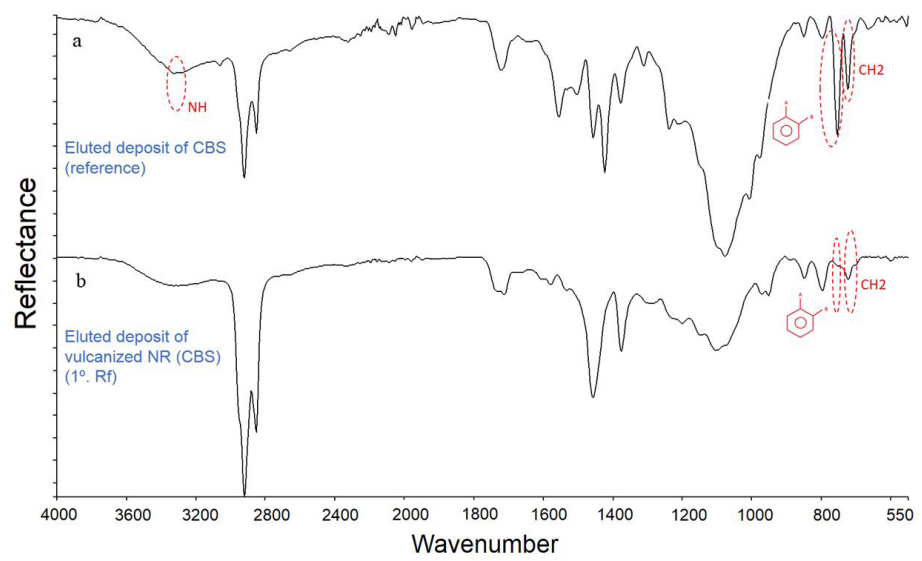

Figure 4. UATR spectra (after TLC, in toluene/acetone (45/65): (a) Eluted deposit of CBS (reference); (b) eluted deposit of vulcanized $\mathrm{NR}(\mathrm{CBS})\left(1^{\circ} . \mathrm{Rf}\right)$.

\subsection{NR (TMTD)}

Despite the fact there would be loss of material and spectral resolution in the TLC/IR technique, perhaps caused by the conditions applied, mainly due to transmission/pellet technique $^{[8]}$ application, Figure 6 shows that in the UATR spectrum of extract, in toluene, of TMTD after TLC, there are very similar absorptions to the reference TMTD UATR spectrum, also to the UATR spectrum of the extract, in toluene/acetone (45/65), which does not happen with the TMTD spectra, obtained in other eluent/developer systems. The Figure suggests that in the analysis of NR spectra, in TLC/UATR technique, the reference spectrum of analyzed accelerator should be used, under the same conditions as the TLC analysis.

By TLC analysis, toluene/ethyl acetate (100:5)/Gibbs that would be used to separate the TMTD, and this can be better visualized by comparing NR(TMTD) $\left(1^{\circ} \mathrm{Rf}\right)$ and TMTD spectra, after TLC, in this system (Figure 7), and with discussion of the spectra being made according to data found in the literature ${ }^{[12]}$.
In recent paper ${ }^{[12]}$, it was evaluated the additives separation, including TMTD, in ethylene propylene diene monomer terpolymer (EPDM), with other eluent systems. It was mentioned that despite the fact there was some overlap of bands, it was possible to indicate in spectra obtained after TLC, in conditions ( $70 \%$ of hexane / $30 \%$ of ethyl ether and $5 \%$ of acetic acid), two absorptions in $1240 \mathrm{~cm}^{-1}(\mathrm{C}=\mathrm{S}, \mathrm{C}-\mathrm{S})$ and $560 \mathrm{~cm}^{-1}(\mathrm{~S}-\mathrm{S})$ of TMTD, also associated to the bands shape of sulfur compounds, even in a small proportion in the formulation (also around 1phr, as in current paper).

In Figure 7, albeit there is also overlap, notably in the region of 3000-2800 and 1400-1460 $\mathrm{cm}^{-1}$, of $\mathrm{CH}_{2}$ and $\mathrm{CH}_{3}$ groups, the $\mathrm{C}-\mathrm{N}$ band, around $1140 \mathrm{~cm}^{-1}$, common bands to other additives, the absorptions of geminated methyl group, between $1380-1350 \mathrm{~cm}^{-1}$ and around $560 \mathrm{~cm}^{-1}$ (S-S), of $\mathrm{TMTD}^{[12,17]}$, as well as their shape, are better visualized in the rubber extract spectra, in comparison with the TMTD spectrum, after TLC, suggesting that the used conditions, toluene / ethyl acetate (100:5) / Gibbs, are suitable for the NR rubber, for this purpose. In addition, the TLC analysis 


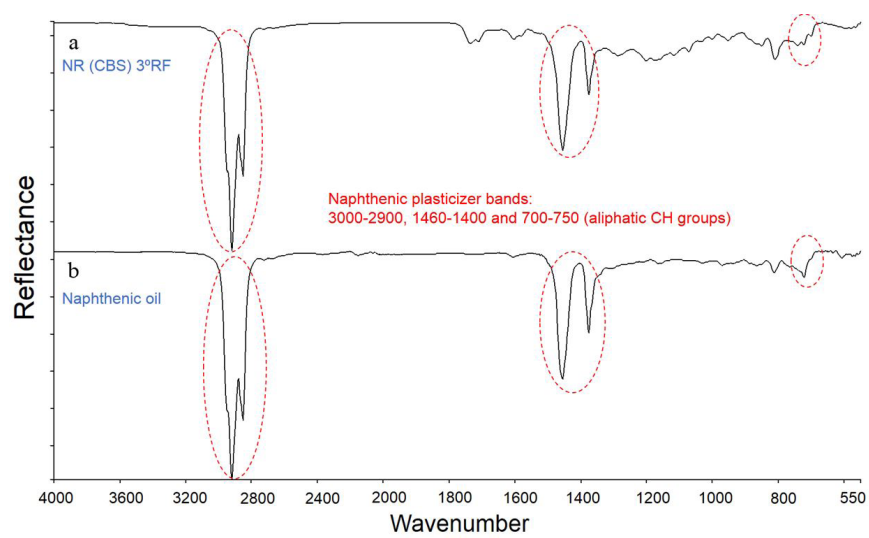

Figure 5. UATR spectra (after TLC): (a) Eluted deposit, in toluene, of vulcanized NR (CBS) ( $3^{\circ}$ Rf); (b) naphthenic oil.

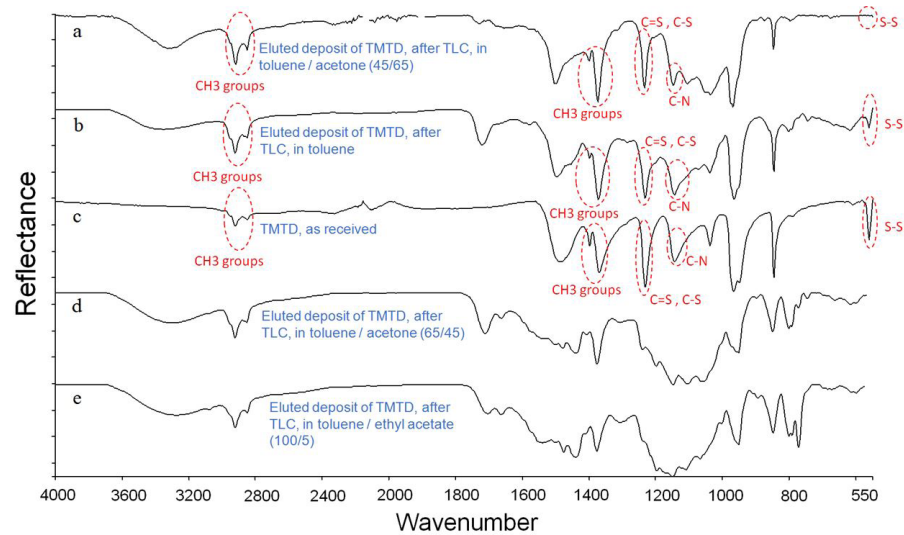

Figure 6. UATR spectra: (a) Eluted deposit of TMTD, after TLC, in toluene/acetone (45/65); (b) Eluted deposit of TMTD, after TLC, in toluene; (c) TMTD, as received; (d) Eluted deposit of TMTD, after TLC, in toluene/acetone (65/45); (e) Eluted deposit of TMTD, after TLC, in toluene/ethyl acetate $(100 / 5)$

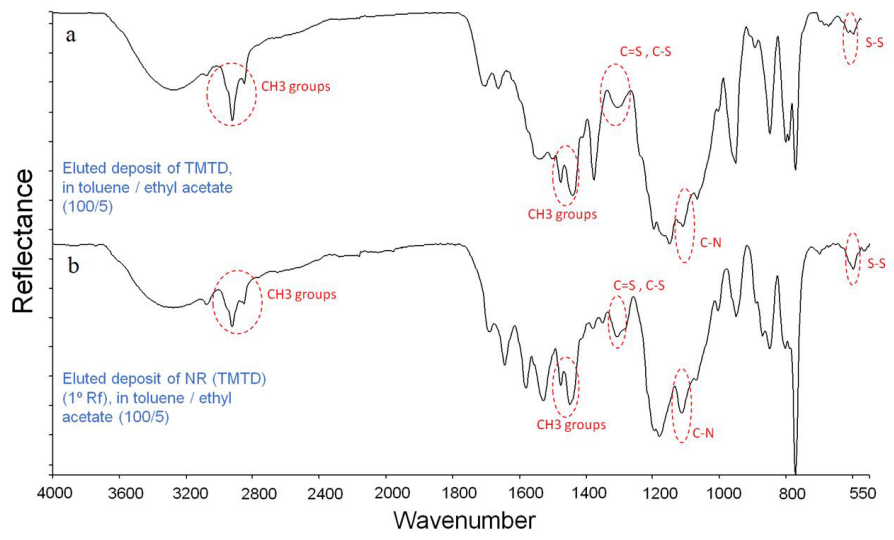

Figure 7. UATR (after TLC) spectra: (a) eluted deposit of TMTD, in toluene/ethyl acetate (100/5); (b) eluted deposit of vulcanized NR (TMTD) ( $1^{\circ}$. Rf), in toluene/ethyl acetate.

showed Rf of the NR(TMTD) rubber extract equal to that observed for the TMTD (reference), indicating the TLC/UATR coupling was effective, as an indication of this accelerator in the formulation.

\subsection{NR (DCP)}

By the TLC analysis, all eluent systems used were efficient for the separation of DCP, with better result for Toluene / Ethyl Acetate (100/5) / Gibbs, $3^{\circ} \mathrm{Rf}$. This is also 


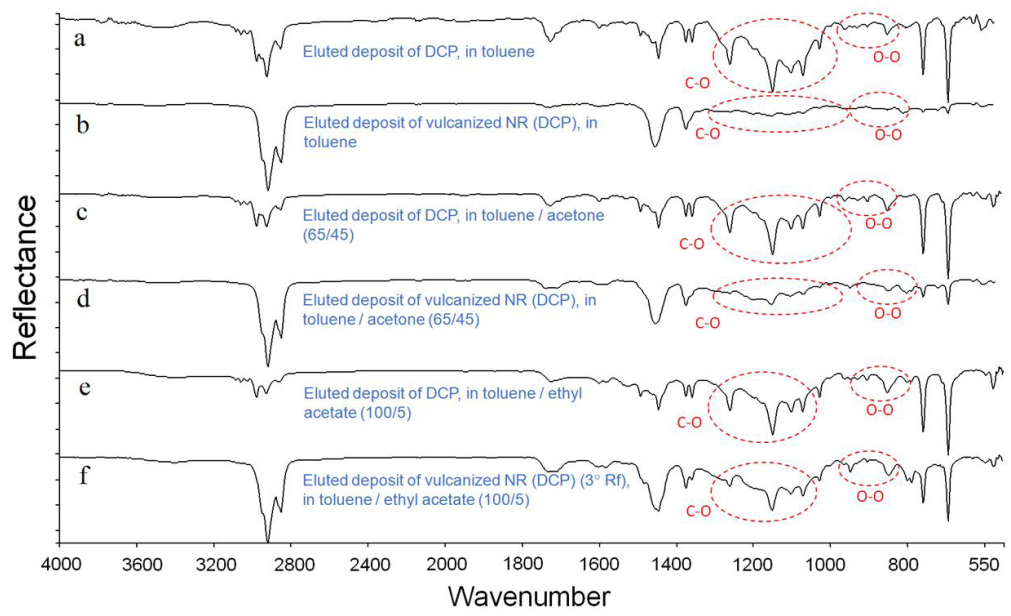

Figure 8. UATR spectra: (a) eluted deposit of DCP, in toluene; (b) eluted deposit of vulcanized NR (DCP), in toluene; (c) eluted deposit of DCP, in toluene/acetone (65/45); (d) eluted deposit of vulcanized NR (DCP), in toluene/acetone (65/45); (e) eluted deposit of DCP, in toluene/ethyl acetate (100/5); (f) eluted deposit of vulcanized NR (DCP) $\left(3^{\circ} \mathrm{Rf}\right)$, in toluene/ethyl acetate (100/5).

demonstrated by the UATR analysis, Figure 8. In this figure, the similarity of the spectra can be noted as well and, with the bands $\left(\mathrm{cm}^{-1}\right)$ being around $1250-1100,980-870$, of variable intensity, weak to strong, assigned to the $\mathrm{C}-\mathrm{O}$ (stronger) and $\mathrm{O}-\mathrm{O}$ (weaker) groups ${ }^{[12]}$, that may fit as reference in the characterization of the DCP presence, which can be better observed, meeting the TLC data.

After these results, the TLC-IR(UATR) analysis applied to separate and characterize NR additives such as CBS, TMTD and DCP, was considered available to rubber quality control in laboratories because is faster and simpler than methodologies involving other coupling types such as $T G / \mathbb{I R}^{[19]}$. In this study ${ }^{[19]}$, although good results were reached for sulfur additives such as MBT and TMTM, they were characterized by a complex methodology of its degradation products. Plasticizer was not detected, whereas in the TLC/UATR showed in this current paper, naphthenic oil was separated and characterized. Degradation studies are not necessary too. However, such methods can be considered complementary, if it necessary wide characterization of rubber additives.

\section{Conclusion}

UATR analysis of acetone NR extracts showed some differences in the formulations, the TMTD presence were better demonstrated. However, there are overlaps of bands of other additives. Therefore, it is necessary a separation/identification step, such as TLC/IR.

By TLC and TLC/UATR analysis, it was only evidenced the CBS separation/identification using the toluene/acetone system (45/65). By TLC/UATR analysis, it was possible to characterize the naphthenic oil presence, with the system (toluene/Gibbs). Perhaps, this separation/identification has been facilitated by this additive, paraffinic oil, being present in a higher proportion in the formulation $(7.7 \mathrm{phr})$ than the other accelerators like CBS and TMTD (5.4 phr) in their specific formulations.
TLC analysis suggested that is achievable to separate TMTD, specifically by the toluene/ethyl acetate (100/5) / Gibbs $\left(1^{\circ} \mathrm{Rf}\right)$ system. TLC/UATR analysis confirmed this indication, despite of some bands overlaps, which was once registered in EPDM rubber formulations, apparently because of the small TMTD amount in referred formulations.

By the TLC/UATR analysis, all eluent systems used were efficient for the separation of DCP, predominantly the Toluene/Ethyl Acetate (100/5)/Gibbs, $3^{\circ} \mathrm{Rf}$, constituting the best methodology result in terms of characterization (TLC) and identification (UATR). It may be explained by the higher quantity of the additive ( $6.4 \mathrm{phr})$ in relation to the others $(1.1 \mathrm{phr})$, in the specific formulations.

The decision to use the same curing time for all formulations did not affect the analysis because the results showed the additives separation by TLC and their identification by FT-IR (UATR), by characteristics bands of DCP (Figure 8) and TMTD (Figure 7). The unambiguous separation and identification of CBS may have been impaired due to the higher content of plasticizer in its formulation, which may have been the factor that facilitated the separation and identification of naphthenic oil (Figure 5).

In short, it was concluded that the developed TLC/UATR methodology for studying the additives not only has it contributed to the potential of formulations characterization in the automotive industry, but also could be applied in the aerospace area. The reason is related to these compounds that can be present in NR and EPDM formulations. It is a multiplier effect of acquired technical knowledge, being extremely useful to predict specific properties for different applications.

\section{Acknowledgements}

This study was supported in part by the National Senior Professor Program (PVNS) from the Coordenação de Aperfeiçoamento Pessoal de Nível Superior (CAPES). 


\section{References}

1. Ciência e Tecnologia da Borracha. Retrieved from in 2017, March 28, from http://ctborracha.com

2. Mohan, C. H. V. R., Ramanathan, J., Kumar, S., \& Gupta, A. V. S. S. K. S. (2011). Characterization of Materials Used in Flex Bearings of Large Solid Rocket Motors. Defence Science Journal, 61(3), 264-269. http://dx.doi.org/10.14429/dsj.61.52.

3. Chemikeys. Retrieved from in 2017, April 18, from http://www. chemkeys.com/blog/wp-content/uploads/2008/09/polimeros. pdf

4. Grinson, E. C. (2010). Borrachas e seus aditivos - Componentes, Influências e Segredos. Porto Alegre: Ed. Letra \& Vida.

5. D’Angelo, A. (2008). Peróxidos resistentes ao oxigênio para vulcanização contínua em túnel de ar quente. Revista Borracha Atual, 1-35. Retrieved in 2017, May 25, from Retrieved from http://www.retilox.com.br/astecnicos/download/peroxidos1. pdf

6. Reyneke-Barnard, C. P., Gradwell, M. H. S., \& Mcgill, W. J. (2000). N,N'Pentamethylenethiuram disulfide- and N,N"Pentamethylenethiuram Hexasulfide-accelerated Sulfur Vulcanization. I. Interaction of Curatives in the Absence of Rubber. Journal of Applied Polymer Science, 77(12), 2718-2731. http:// dx.doi.org/10.1002/1097-4628(20000919)77:12<2718::AIDAPP200>3.0.CO;2-E

7. Joseph, A. M., George, B., Madhusoodanan, K. N., \& Alex, R. (2015). Current status of sulphur vulcanization and devulcanization chemistry: Process of vulcanization. Rubber Science, 28(1), 82-121.

8. Charoeythornkhajhornchai, P., Samthong, C., \& Somwangthanaroj, A. (2017). Influence of sulfenamide accelerators on cure kinetics and properties of natural rubber foam. Journal of Applied Polymer Science, 134(19), 44822. http://dx.doi.org/10.1002/ app.44822.

9. Kruželák, J., Sýkora, R., \& Hudec, I. (2014). Peroxide vulcanization of natural rubber. Part I: effect of temperature and peroxide concentration. Journal of Polymer Engineering, 34(7), 617-624.
10. Dutra, R. C. L. (1996). Aplicação de técnica de TLC-IR em estudos de separação, identificação e quantificação de aditivos em borrachas. Polímeros: Ciência e Tecnologia, 6(2), 26-31.

11. Rodrigues, V. C., Diniz, M. F., Mattos, E. C., \& Dutra, R. C. L. (2016). Separação e identificação de aditivos em tinta por TLC-IR/ UATR e extração seletiva. Polímeros: Ciência e Tecnologia, 26(Special Number), 68-74.

12. Damazio, D., Campos, E. A., Diniz, M. F., Mattos, E. C., \& Dutra, R. C. L. (2016). TLC/IR (UATR) off-line coupling for the characterization of additives in EPDM rubber compositions. Polímeros: Ciência e Tecnologia, 26(1), 74-80.

13. Chauveau, S., Hamon, M., \& Leleu, E. (1991). Separation and identification of various vulcanization agents and antioxidants in two types of rubber by chromatographic and spectrometric methods. Talanta, 38(11), 1279-1283. http:// dx.doi.org/10.1016/0039-9140(91)80106-A. PMid:18965298.

14. Sanches, N. B., Cassu, S. N., Diniz, M. F., \& Dutra, R. C. L. (2014). Characterization of additives typically employed in EPDM formulations by using FT-IR of gaseous pyrolyzates. Polímeros: Ciência e Tecnologia, 24(3), 269-275.

15. Mueller, D., Ferrão, M. F., Marder, L., Costa, A. B., \& Schneider, R. C. S. (2013). Fourier Transform Infrared Spectroscopy (FTIR) and multivariate analysis for identification of different vegetable oils used in biodiesel production. Sensors, 13(4), 4258-4271. http://dx.doi.org/10.3390/s130404258. PMid:23539030.

16. Wake, W. C., Tidd, B. K., \& Loadman, M. J. R. (1983). Analysis of rubber and rubber-like polymer (3rd ed.). New York: Applied Science.

17. Smith, A. L. (1979). Applied infrared spectroscopy. New York: John Wiley \& Sons.

18. Wolfang, W. (1987). Tópicos de espectroscopia no infravermelho. São José dos Campos: ITA.

19. Sanches, N. B., Cassu, S. N., Diniz, M. F., \& Dutra, R. C. L. (2015). TG/FT-IR characterization of additives typically employed in EPDM formulations. Polímeros: Ciência e Tecnologia, 25(3), 247-255.

Received: June 20, 2017 Revised: Sept. 28, 2017 Accepted: Dec. 07, 2017 